\title{
Early intervention with ABA prevents neuroinflammation and memory impairment in a triple transgenic mice model of Alzheimer's disease
}

Verónica Espinosa-Fernández ${ }^{1 \#}$, Aroa Mañas-Ojeda ${ }^{1 \#}$, Mar Pacheco-Herrero², Ernestina Castro-

$$
\text { Salazar }^{1,2,} \text { Francisco Ros-Bernal }{ }^{1} \text {, Ana María Sánchez-Pérez }{ }^{1 *}
$$

${ }^{1}$ Department of Medicine, University of Jaume I, Castellón de la Plana, Spain

${ }^{2}$ Department of Medicine, Pontificia Universidad Católica Madre y Maestra. República Dominicana \# Equal contribution

*Correspondence to

Ana María Sánchez-Pérez, email: sanchean@ uji.es. Tel +34964387759

ORCID 0000-0002-5811-0005

Keywords: Hippocampus, Prefrontal cortex, Neuroinflammation, Microglia morphology, phytohormones.

\section{Acknowledgements}

This work was supported by Plan Propi UJI-B2018-01 to AMSP, and by FONDOCyT (Dominican Republic) to MPH. The authors want to thank the generous donations from the Association of Alzheimer Families, AFA and Salomé Moliner Fundatión, Castellon (Spain).

\section{Highlights}

- Abscisic Acid (ABA) treatment can effectively prevent memory impairment in a murine model of Alzheimer disease (AD).

- ABA treatment can prevent microglia transition to inflammatory state in transgenic model of AD.

- The beneficial effects of ABA, PPAR $\gamma$ agonist and an insulin sensitizer in the central nervous system are independent of peripheral insulin resistance.

- Further studies will establish whether later intervention (when the disease may be in initial stages), but longer treatments can guarantee better rescue of memory impairment. 


\title{
Abbreviations
}

ABA, Abscisic acid;

$\mathrm{AD}$, Alzheimer disease

APP, Amyloid precursor protein;

PFA, paraformaldehyde

PPAR- $\gamma$, Peroxisome proliferator-activated receptor gamma;

SEM, Standard error of mean.

TNF $\alpha$, Tumour necrosis Factor $\alpha$

IL1 $\beta$, Interleukin

VEH, vehicle

\begin{abstract}
Neuroinflammation and insulin resistance in the brain are intimately linked to neurodegenerative disorders, including Alzheimer's disease. Even though traditionally Alzheimer's disease has been associated to A $\beta$ deposits and hyperphosphorylated Tau intracellular tangles, several studies show that neuroinflammation may be the initial cause that triggers degeneration. Accordingly, a number of natural supplements that improves brain insulin sensitivity and reduce neuroinflammation have been proposed as good choices in the therapeutic prevention of cognitive decline. Further supporting this evidence, we show that phytohormone Abscisic Acid, can prevent memory impairment and neuroinflammation markers in a triple transgenic mouse model, where no peripheral inflammatory changes have occurred. Moreover, our data strongly suggests that early intervention is critical for good prognosis, and that cognitive improvement requires longer treatment than recovering neuroinflammation markers.
\end{abstract}

\section{Main text}

A large body of evidence has accumulated correlating chronic inflammatory processes that results in neuronal toxicity and the development of neurodegenerative pathologies [1], including Alzheimer's disease $[2,3]$. In line with this, several extrinsic factors such as physical exercise, supplementary dietary compounds and relaxing methods exert their beneficial effects on brain most likely due to their capability of reducing chronic and sustained inflammation. Consequently, synaptic plasticity [4] and memory [5, 6] are greatly ameliorated. Neuroinflammation is characterized by an important increase of reactive microglia, which secretes proinflammatory cytokines (e.g. TNF $\alpha$, IL1 $\beta$ ). Sustained high levels of these cytokines result in brain insulin resistance and further neuronal toxicity. Preventing microglia inflammatory state has been associated to improved spatial memory [7]. Thus, phytohormones can be considered neuroprotectors due to their anti-inflammatory properties [8]. Previous work in our group lab have shown that, ABA, a 
PPARP $\gamma$ agonist [9] can improve glucose tolerance in obesity models, reduce neuroinflammation and restore cognitive function in a HFD-induced neuroinflammation model [10-13].

We hypothesized that ABA could have a potential beneficial effect in a genetic model of neuroinflammation, unrelated to obesity or peripheral insulin resistance. In this study, we have made use of a well-established model of synaptic dysfunction, the triple transgenic mice (3xTg-AD) to evaluate the effects of the phytohormone $\mathrm{ABA}$ in hippocampal-dependent cognitive tasks, in correlation with neuroinflammation processes. This murine model of Alzheimer is homozygous for all three mutant alleles (Psen1 mutation; APPSwe and tauP301L) transgenes. These are based on human mutations identified in Familial AD [14]. The procedures followed directive 86/609/EEC of the European Community on the protection of animals used for experimental and other scientific purposes. The experiments were approved by the Ethics Committee of the University Jaume I (approval number 2014/VSC/PEA00209).

Control and transgenic mice (total of 78) were divided randomly into four experimental groups: ABA3 and ABA5, animals supplemented with ABA (Fernandez-Rapado, Spain) in their drinking water (20 mg/L) for 3 or 5 months respectively. Control groups where administered vehicle for 3 or 5 months (VEH3 or VEH5 respectively). The number of animals per group was 8-10. All groups were fed ad libitum for 8 months. At that age, all experimental groups were subjected to behavioural tests prior to sacrifice (Fig 1).

The Novel Object Recognition (NOR) paradigm exploits the innate preference exhibited by rodents to explore novel objects. This paradigm evaluates the capability of the animal to remember a familiar object compared to a new one. We observed no significant differences in the time spent exploring the identical objects during familiarization phase (Fig. 2A). When an object was substituted for a new one (test phase), control mice spent $71 \pm 0.03 \%$ or $72 \pm 0.05 \%$ of the total test time sniffing the new object (VEH 3 and VEH 5 respectively). On the other hand, transgenic mice spent $53 \pm 0.05 \%$ and $58 \pm 0.05 \%$ of the total test time sniffing the novel object, which indicates that transgenic mice do not remember the familiar as well and thus explore both objects almost equally. When mice were treated with ABA, controls behaviour did not change respect to their vehicle-administered littermates ( $73 \pm 0.07 \%$ and $70 \pm 0.03 \%$ respectively). Triple transgenic mice had a slight improvement in memory after 3months of ABA treatment $(66 \pm 0.1 \%)$ but this increase was not significant. However, 5 months ABA treatment completely restored memory in transgenic mice, that behaved as controls (76 $\pm 0.06 \%$ time exploring the novel object) (Fig. 2B) ${ }^{* *}$ p $<0.01$ respect to control and ${ }^{\# \#} \mathrm{p}<0.01$ respect to $3 \mathrm{xTg}$ VEH5. Data are expressed as the percentage of the total time of the test that the animal spends sniffing the novel object. Data passed normality test and was analysed with unpaired Student t-test for differences. Moreover, transgenic mice at this age did not differ from controls in social preference, nor did ABA treatment affect this behaviour (Fig 2C). Moreover, social memory was not different either in transgenic versus control mice and treatment did not affect this memory at this age (Fig. 2D). 
In this study we confirmed that short-term memory was deficient in transgenic mice compared to controls, whereas social discrimination and social memory were no different from controls. This could be consistent with an early sign of hippocampal dependent task in Alzheimer patients and different of other dementias [15]. We found that the longest treatment with ABA (5 months) but not short term (3 months) completely rescued object recognition impairment. This finding could be explained either because the effect is due to early the intervention (the long treatment started when the mice were only 3 month of age), before irreversible neurological damage has occurred. The other, more hopeful alternative is that longer treatment is required to effectively reverse the toxic consequences of Alzheimer-inducing mutations. Further experimental designs will be required to elucidate between these possibilities.

Microglia morphology has been demonstrated to correlate with their activation state [16]. Thus, in healthy conditions, the microglia display a branched morphology. Although these microglia cells are called "resting. microglia, they are actually in a surveillant activity, where branches are used to scrutinize the environment and regulate neuronal activity [17]. In inflammatory conditions, or after any kind of injury, microglia undergo a stepwise de-ramification process, acquiring a more rounded morphology, with fewer branches. In this morphological state, microglia are known as pro-inflammatory or "reactive". Both microglia phenotypes display a distinct pattern of cytokines secretion. Several studies have validated a quantification method to evaluate the microglia morphology and transitions from resting to reactive inflammatory state $[18,19]$.

The murine models of Alzheimer disease exhibit microglia in an inflammatory state, mostly due to the accumulation of toxic misfolded proteins. Therefore, we set out to examine the microglia morphology in two brain areas that are involved in memory, the prefrontal cortex and the hippocampus). To evaluate microglia, immunodetection of Iba-1, microglia marker was carried out as described [10-13]. Briefly, mice were anesthetized with pentobarbital (120 mg/kg Eutanax, Fatro, Barcelona, Spain) and transcardially perfused with saline and $4 \%$ paraformaldehyde (PFA) fixative. Sliding Microtome Leica SM2010R (Leica Microsystems, Heidelberg, Germany) was used to obtain $40-\mu \mathrm{m}$ thick coronal frozen sections. Sections were rinsed and incubated in blocking solution for $1 \mathrm{~h}$ followed by primary antibody incubation anti-IBA1 (ab5076; Abcam 1:500). After several washes, the slides were incubated with the biotinylated secondary antibody (Jackson 1: 200). Signal was detected using the VECTASTAIN® Elite ${ }^{\circ}$ ABC-HRP Kit; Peroxidase, Standard, USA) and colour reaction with DAB (Sigma-Aldrich, St Louis, MO, USA) for 1520 minutes. The slices were and cover-slipped with a DPX mounting medium.

A total of 5 cells per brain area (prefrontal cortex and/or hippocampus) were randomly selected in each animal per experimental groups $(n=4)$. The following microglia morphological parameters were analysed with FIJI free software: fractal dimension (D); this parameter evaluates cellular branching complexity. High D values correspond to a greater cellular complexity; cell area is the total number of pixels 
corresponding to the area occupied by the cell, soma and branches, thus the higher the area the microglia is considered in resting state. Finally, cell perimeter is measured based on the single outline cell shape, thus, the higher the ramification, the higher this value and the microglia is considered polarized into a less inflammatory state $[18,19]$. Data are expressed as mean \pm SEM of the 4 animals and subjected to a Kolmogorov-Smirnov normality test. Since the sample was small, Mann Whitney non-parametric test was applied.

We observed that transgenic mice treated with vehicle had clear inflammatory microglia compared to control mice, both in hippocampus (Fig 3) and in prefrontal cortex (Fig 4) areas. Interestingly, both shortand long-term ABA treatment prevented the inflammatory conversion of microglia, in both areas, as demonstrated by the quantification of specific parameters. Fractal, area and perimeter are statistically different between control and 3xTg (treated with VEH 3 and VEH 5); whereas transgenic mice treated with ABA 3 and ABA5 showed no difference with their respective controls. Furthermore, 3xTg ABA3 and ABA5 were significantly different from $3 x T g$ vehicle treated experimental groups. ${ }^{*}<<0.05$ indicates that $3 \times \mathrm{xg} \mathrm{VEH}$ is significantly different of control VEH; whereas ${ }^{\#} \mathrm{p}<0.05$ indicates that $3 \mathrm{xTg} \mathrm{ABA}$ is significantly different of 3xTg VEH.

ABA targets peroxisome proliferator-activated receptor gamma (PPAR- $\gamma$ ) in a similar manner as the thiazolidinedione class of anti-diabetic drugs [20] and curcumin [21]. This family of PPAR- $\gamma$ agonist molecules has been proposed as a new class of therapeutic molecules to treat central nervous system disorders [22]. Consistently, we have demonstrated the beneficial effects of ABA in a neuroinflammation model induced by high fat diet [10, 13].

Whether or not ABA activates microglia has been controversial. In vitro studies have shown that ABA may induce activation of microglial cell line N9 via calcium influx [23], where others have not confirmed this activation [24]. In our model, we have clearly demonstrated that ABA chronic exposure (3 and 5 months) can effectively reduce (or prevent) microglia chronic activation as measured by morphological parameters of perimeter, area and fractal dimension. However, this reduction in proinflammatory state does not totally correlate with improvement in spatial memory, since 3 months ABA exposure effectively prevented microglia conversion to inflammatory state, but did not rescue behaviour. This suggests that microglia state is not enough in itself to guarantee optimal neuronal performance, but it is very likely an early event, prior to the improvement of neuronal health, and subsequent optimal cognitive task performance. In addition, ABA does not affect controls, suggesting that is a safe procedure.

Considering all these data, we conclude that ABA can effectively prevent memory deficits in a model of neuroinflammation induced by Alzheimer-like mutations, supporting the beneficial effects on memory that we have reported previously in a model of neuroinflammation due to high fat diet. These data broad up the applications of safe and natural anti-inflammatory supplements in a variety of pathologies mediated but 
chronic inflammation, especially in the elderly. In addition, we show that early intervention may be crucial and therefore prompt for the early detection to prevent neurological irreversible damage with better chances of delaying or avoiding disease, even in the hereditary forms of the disease.

In conclusion in this study, we have evaluated the effects of ABA chronic short (3 months) and long for (5 months) treatment. All mice were sacrificed at 8 months, which means that long treatment started in young adult animals, before the neurological damage is suspected in this model. We have shown that the long but not the short treatment can prevent memory deficits. However, to our surprise, ABA both long and short term administration prevented hippocampal and prefrontal cortex microglia inflammatory state, suggesting that microglia resting state is not sufficient for optimal cognitive function, but it probably precedes it. Our results indicate that $\mathrm{ABA}$ can be an effective supplement to improve cognitive health in neuropathologic situations. Moreover, early intervention and/or longer treatments may be critical to prevent cognitive decline.

Figure 1. Experimental design. $3 x \mathrm{Tg}$ and C57BL/6 male mice were treated with ABA (20mg/L) or vehicle in the drinking water. Treatment was administered for 5months (VEH 5M or ABA 5M), starting at 3 months of age; and 3months (VEH $3 \mathrm{M}$ or ABA $3 \mathrm{M}$ ), starting at 5 months of age. A week prior to sacrifice all groups performed behavioural tests as depicted. All groups were sacrificed at 8 months of age.

Figure 2. ABA chronic administration improves memory in triple transgenic mice. Novel object recognition paradigm, time exploring two objects during familiarization phase is similar in all groups (A). Time exploring the novel object during test phase is lower in $3 \times \mathrm{Tg}$ but recover with 5months ABA treatment (B). In a three-chamber social interaction and memory test, Sociability (C) nor Social memory was altered by genotype or ABA treatment (D). Data expressed as the mean and SEM of $\mathrm{n}=6-10$ individuals ${ }^{*} \mathrm{p}<0.05 ;{ }^{*} \mathrm{p}<<0.01$ respect to control; ${ }^{\#} \mathrm{p}<0.01$ respect to $3 \mathrm{xTg}$ VEH5 (one tail Student t-test).

Figure 3. Microglia in hippocampus CA1. (A) Microglia from transgenic mice with 3 and 5 month vehicle treatment shows a proinflammatory morphology compared to wild type control. ABA treatment for 5 and 3 months rescues microglia morpholgy in transgenic mice and does not alter control. (B) Quantification of morphological characteristics showed that fractal, area and perimeter characteristics are lower in vehicle treated transgenic mice compared to control. ABA administration for 3 and 5 months rescued microglia morpholgy to control parameters while does not significantly affect wild type mice. Data expressed as the mean and SEM of 4 animals per group (5 cells per Bregma level). Control (black squares) and 3xTg (white circles). ${ }^{*} \mathrm{p}<0.05$ significant difference 
3xTg3 VEH versus control VEH ; " $\mathrm{p}<0.053 \mathrm{xTg}$ ABA versus 3xTg VEH (Mann Whitney test).

Figure 4. Microglia in Prefrontal cortex. (A) Microglia from transgenic mice with 3 and 5 month vehicle treatment shows a proinflammatory morphology compared to wild type control. ABA treatment for 5 and 3 months rescues microglia morpholgy in transgenic mice and does not alter control. (B) Quantification of morphological characteristics showed that fractal, area and perimeter characteristics are lower in vehicle treated transgenic mice compared to control. ABA administration for 3 and 5 months rescued microglia morphology to control parameters while does not significantly affect wild type mice. Data expressed as the mean and SEM of 4 animals per group (5 cells per Bregma level). Control (black squares) and 3xTg (white circles). ${ }^{*} \mathrm{p}<0.05$ significant difference 3xTg3 VEH versus control VEH ; " $p<0.05$ 3xTg ABA versus 3xTg VEH (Mann Whitney test).

\section{Bibliography}

1. Stephenson J, Nutma E, van der Valk P, Amor S (2018) Inflammation in CNS neurodegenerative diseases. Immunology 154:204-219. https://doi.org/10.1111/imm.12922

2. Breitner JCS (1996) THE ROLE OF ANTI-INFLAMMATORY DRUGS IN THE PREVENTION AND TREATMENT OF ALZHEIMER'S DISEASE

3. Heneka MT, Carson MJ, Khoury J El, et al (2015) Neuroinflammation in Alzheimer's disease. Lancet Neurol 14:388-405. https://doi.org/10.1016/S1474-4422(15)70016-5

4. Cobain MR, Foreyt JP (2005) Neurobiology of Aging 26S (2005) S85-S87 Designing \&quot;lifestyle interventions\&quot; with the brain in mind. https://doi.org/10.1016/j.neurobiolaging.2005.09.015

5. Praag H Van, Kempermann G, Gage FH (1999) van Praag et al., 1999 - Running increases cell proliferation and neurogenesis in the adult mouse dendate gyrus. Nat Neurosci 2:266-270. https://doi.org/10.1038/6368

6. Vivar C, Potter MC, Praag H Van, van Praag H (2013) All about running: synaptic plasticity, growth factors and adult hippocampal neurogenesis. Curr Top Behav Neurosci 15:189-210. https://doi.org/10.1007/7854

7. Xiong JY, Li SC, Sun YX, et al (2015) Long-term treadmill exercise improves spatial memory of male APPswe/PS1dE9 mice by regulation of BDNF expression and microglia activation. Biol Sport 32:295-300. https://doi.org/10.5604/20831862.1163692

8. Sawikr Y, Yarla NS, Peluso I, et al (2017) Neuroinflammation in Alzheimer's Disease: The Preventive and Therapeutic Potential of Polyphenolic Nutraceuticals, 1st ed. Elsevier Inc. 
9. Bassaganya-Riera J, Guri AJ, Lu P, et al (2011) Abscisic acid regulates inflammation via ligandbinding domain-independent activation of peroxisome proliferator-activated receptor gamma. $\mathrm{J}$ Biol Chem 286:2504-16. https://doi.org/10.1074/jbc.M110.160077

10. Sánchez-Sarasúa S, Moustafa S, García-Avilés Á, et al (2016) The effect of abscisic acid chronic treatment on neuroinflammatory markers and memory in a rat model of high-fat diet induced neuroinflammation. Nutr Metab (Lond) 13:73. https://doi.org/10.1186/s12986-016-0137-3

11. Magnone M, Ameri P, Salis A, et al (2015) Microgram amounts of abscisic acid in fruit extracts improve glucose tolerance and reduce insulinemia in rats and in humans. FASEB J 29:4783-4793. https://doi.org/10.1096/fj.15-277731

12. Qi C-C, Ge J-F, Zhou J-N (2015) Preliminary evidence that abscisic acid improves spatial memory in rats. Physiol Behav 139:231-239. https://doi.org/10.1016/J.PHYSBEH.2014.11.053

13. Ribes-Navarro A, Atef M, Sánchez-Sarasúa S, et al (2019) Abscisic Acid Supplementation Rescues High Fat Diet-Induced Alterations in Hippocampal Inflammation and IRSs Expression. Mol Neurobiol 56:454-464. https://doi.org/10.1007/s12035-018-1091-Z

14. Sterniczuk R, Antle MC, LaFerla FM, Dyck RH (2010) Characterization of the 3xTg-AD mouse model of Alzheimer's disease: Part 2. Behavioral and cognitive changes. Brain Res 1348:149-155. https://doi.org/10.1016/J.BRAINRES.2010.06.011

15. Yew B, Alladi S, Shailaja M, et al (2012) Lost and Forgotten? Orientation Versus Memory in Alzheimer's Disease and Frontotemporal Dementia. J Alzheimer's Dis 33:473-481. https://doi.org/10.3233/JAD-2012-120769

16. Davis EJ, Foster TD, Thomas WE (1994) Cellular forms and functions of brain microglia. Brain Res Bull 34:73-78. https://doi.org/10.1016/0361-9230(94)90189-9

17. Nimmerjahn A, Kirchhoff F, Helmchen F (2005) Resting microglial cells are highly dynamic surveillants of brain parenchyma in vivo. Science 308:1314-8.

https://doi.org/10.1126/science.1110647

18. Fernández-Arjona M del M, Grondona JM, Granados-Durán P, et al (2017) Microglia Morphological Categorization in a Rat Model of Neuroinflammation by Hierarchical Cluster and Principal Components Analysis. Front Cell Neurosci 11:235. https://doi.org/10.3389/fncel.2017.00235

19. Young K, Morrison H (2018) Quantifying Microglia Morphology from Photomicrographs of Immunohistochemistry Prepared Tissue Using ImageJ. J Vis Exp 1-9. https://doi.org/10.3791/57648

20. Guri AJ, Hontecillas R, Ferrer G, et al (2008) Loss of PPAR $\gamma$ in immune cells impairs the ability of abscisic acid to improve insulin sensitivity by suppressing monocyte chemoattractant protein-1 
expression and macrophage infiltration into white adipose tissue. J Nutr Biochem 19:216-228. https://doi.org/10.1016/J.JNUTBIO.2007.02.010

21. Lin J, Chen A (2008) Activation of peroxisome proliferator-activated receptor- $\gamma$ by curcumin blocks the signaling pathways for PDGF and EGF in hepatic stellate cells. Lab Investig 88:529540. https://doi.org/10.1038/labinvest.2008.20

22. Bernardo A, Minghetti L (2006) PPAR- $\gamma$ Agonists as Regulators of Microglial Activation and Brain Inflammation. Curr Pharm Des 12:93-109. https://doi.org/10.2174/138161206780574579

23. Bodrato N, Franco L, Fresia C, et al (2009) Abscisic Acid Activates the Murine Microglial Cell Line N9 through the Second Messenger Cyclic ADP-ribose. J Biol Chem 284:14777-14787. https://doi.org/10.1074/jbc.M802604200

24. Jiang SX, Benson CL, Zaharia LI, et al (2010) Abscisic acid does not evoke calcium influx in murine primary microglia and immortalised murine microglial BV-2 and N9 cells. Biochem Biophys Res Commun 401:435-439. https://doi.org/10.1016/J.BBRC.2010.09.074 


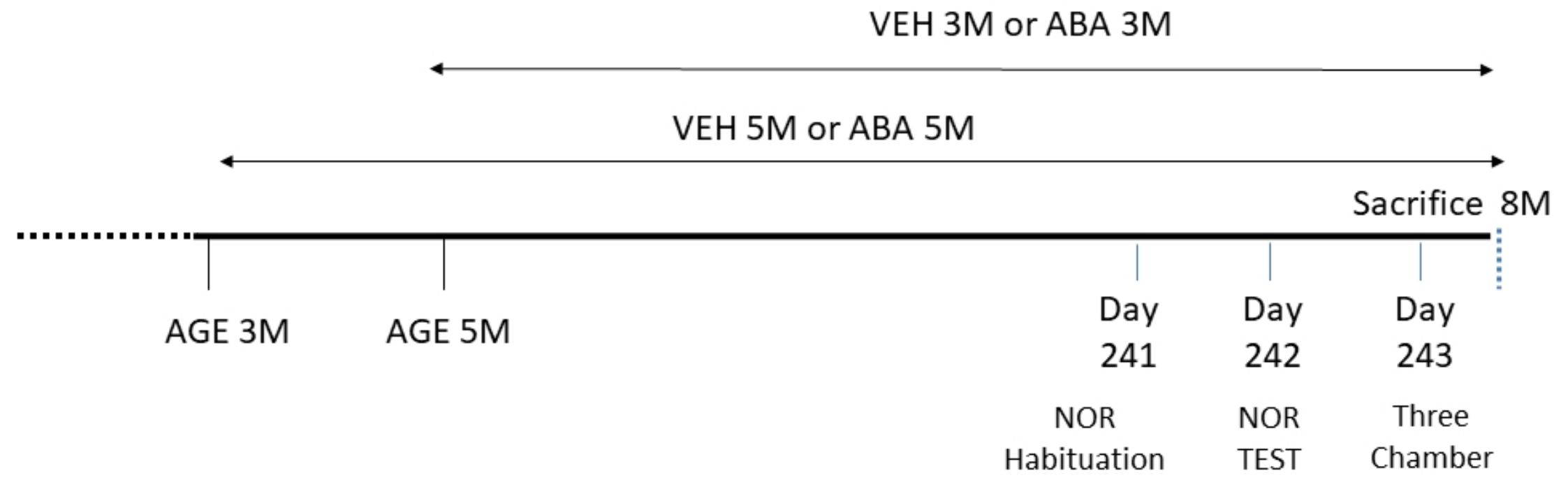



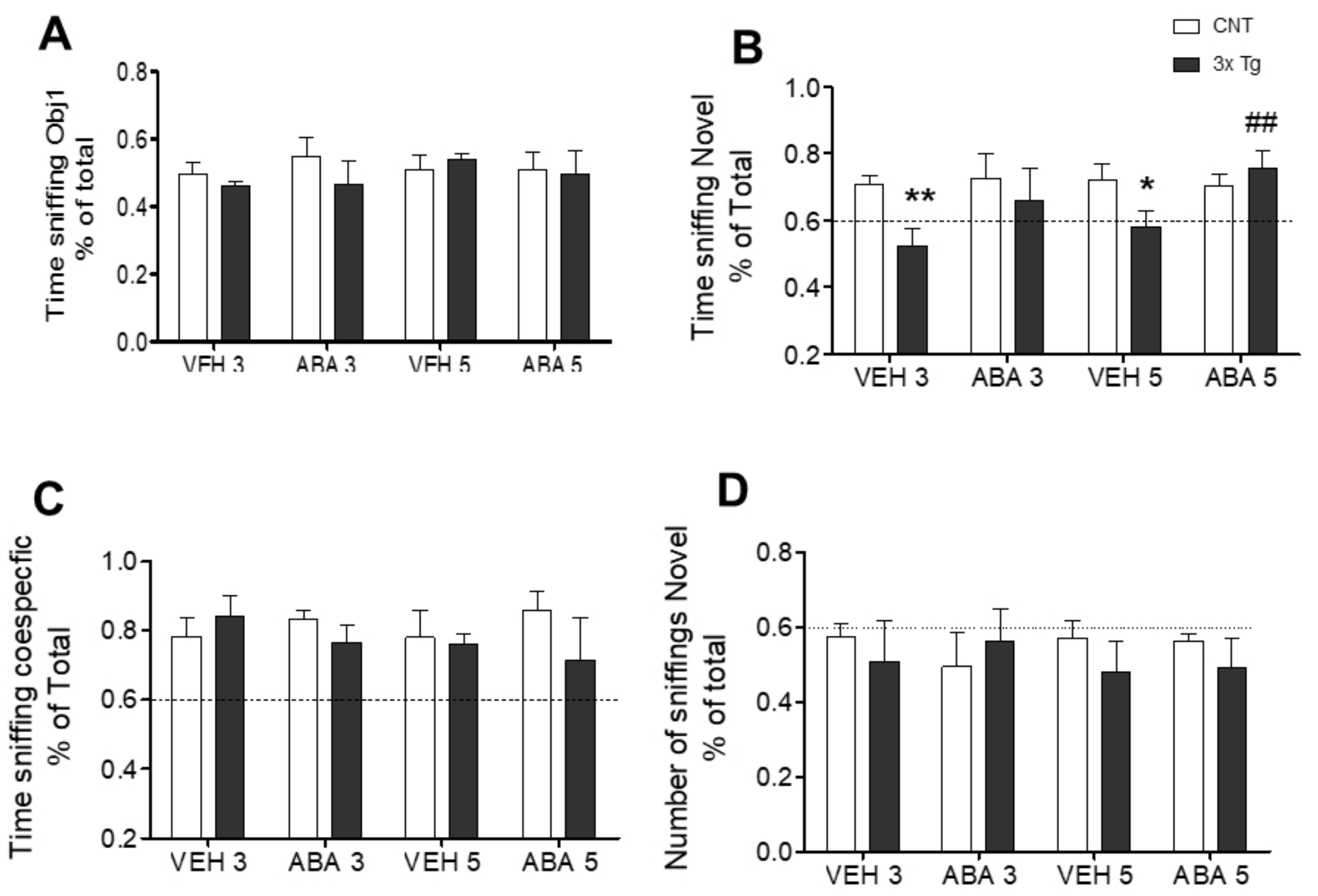
A
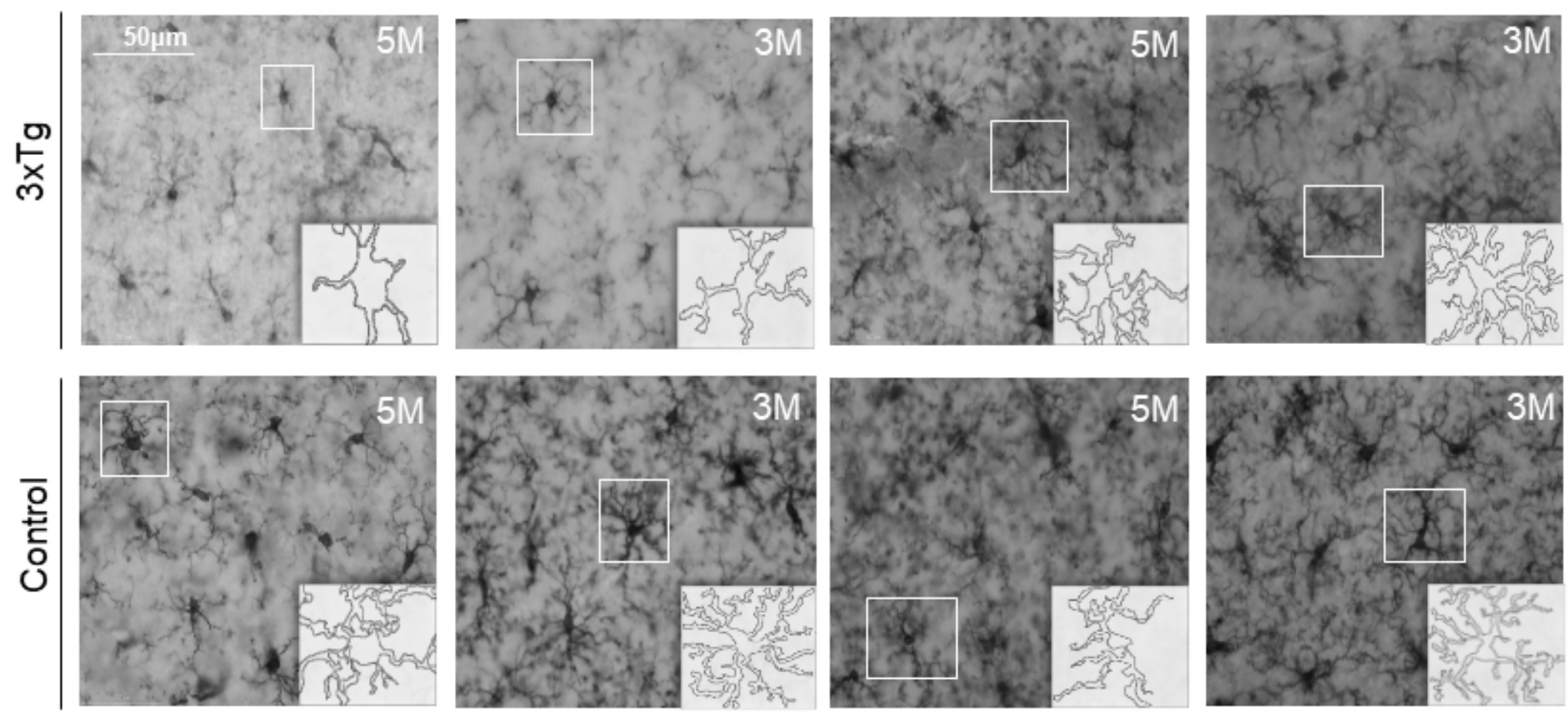

B

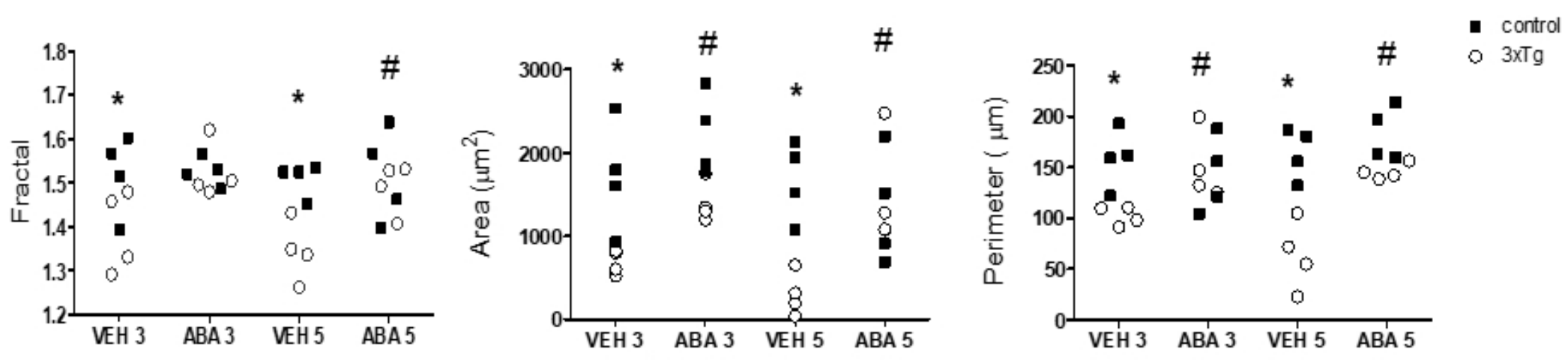


VEH

A
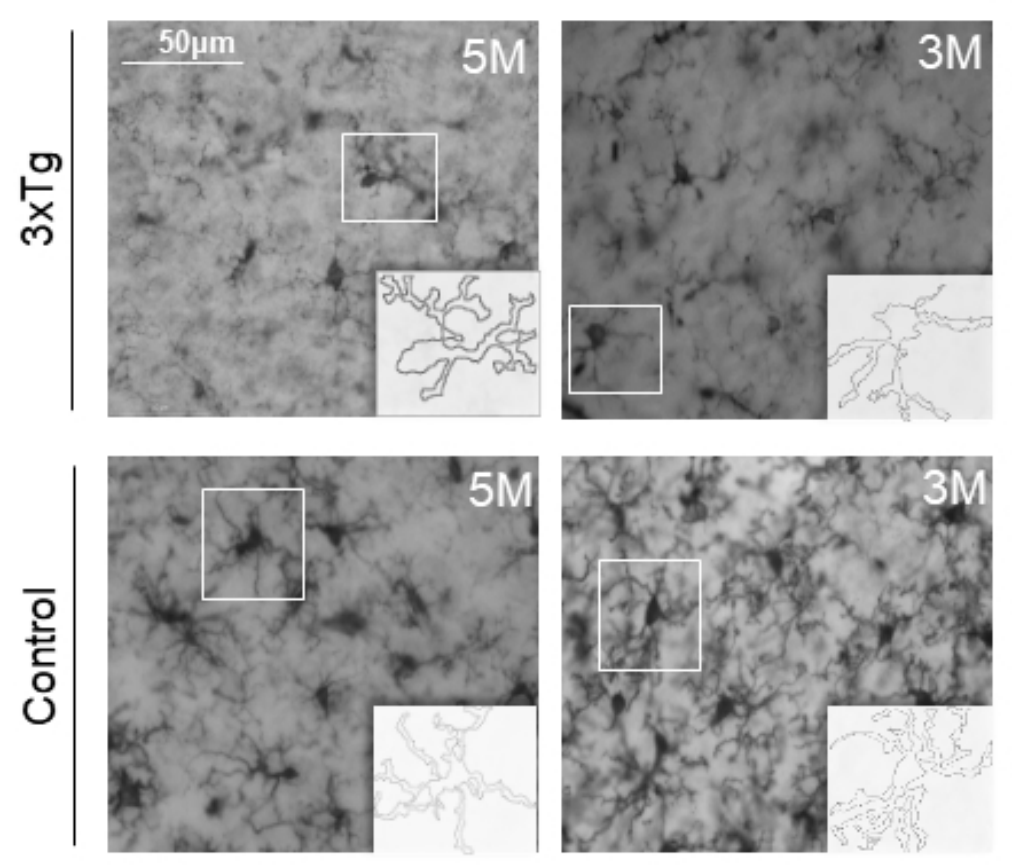

ABA
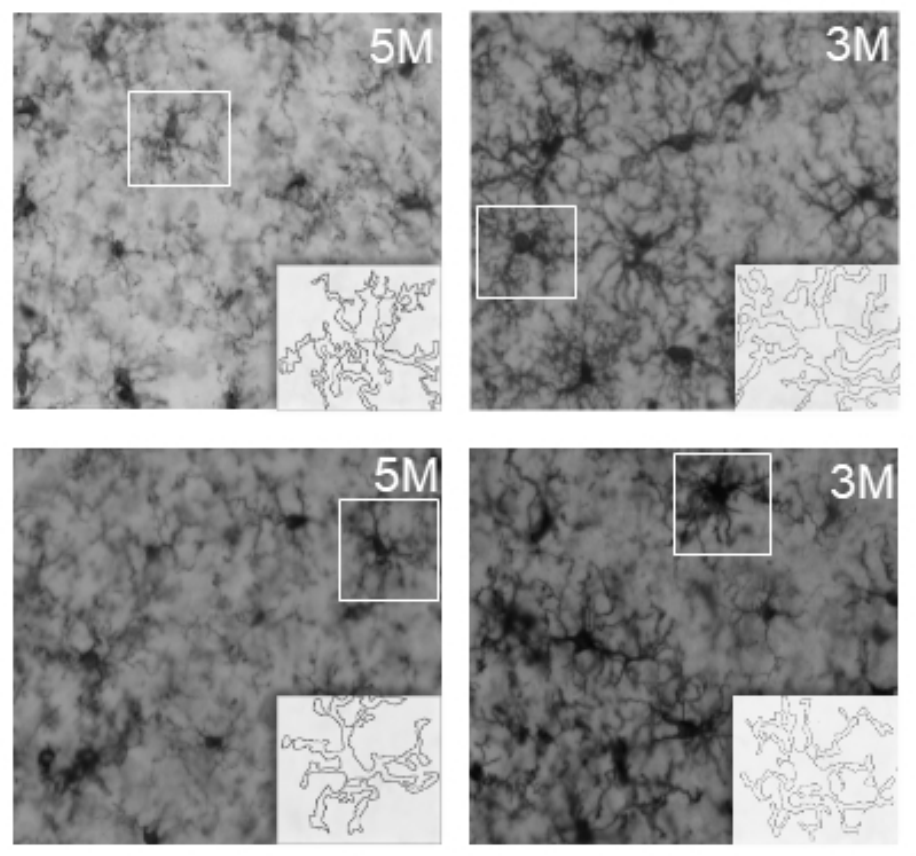

B

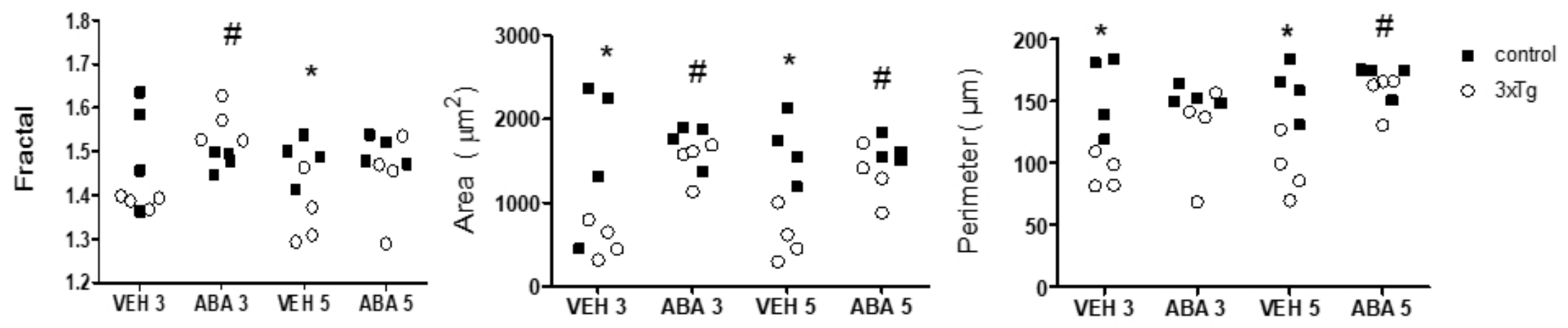

